\title{
Control Study of the Harvesting Robot in Virtual Environment
}

\author{
Haixin Zou ${ }^{1,2}$, Zhumei Song ${ }^{1, *}$, Xiangjun Zou ${ }^{2, *}$, Rong Huang ${ }^{1}$, and Juntao Xiong ${ }^{2}$ \\ ${ }^{1}$ Shenzhen Institute of Information Technology, Shenzhen 518172, china \\ ${ }^{2}$ Key Laboratory of Key Technology on Agricultural Machine and Equipment (South China Agricultural University), Ministry of \\ Education China, Guangzhou 510642, china \\ ${ }^{*}$ Corresponding author
}

\begin{abstract}
Aiming at the challenge of intelligent control of harvesting robot, this study firstly introduced the concept of the harvesting behavior and its basic behaviors, and based on those concepts the behavior knowledge database was established as a library of the functions of the basic behaviors. Secondly, a model of behavior control was proposed based on the vision sensors, and the model includes the weight coefficients $\omega$ and the time sequence coefficient $T$ which could provide the possibility of combining the different basic behaviors in the behavior knowledge database. Thirdly, an intelligent simulation system of the behavior control was developed. Finally, preliminary tests in laboratory were carried out to testify the simulation system and the control system.
\end{abstract}

Keyword-harvesting robot; behavior control; intelligent simulation system

\section{INTRODUCTION}

China is a large traditional agricultural country producing a huge amount of fruit and vegetable in the world. However, many harvesting jobs are still done with hands or simple tools $[1,2]$. Therefore, machines or robots are desired to do the harvesting job. Researchers have been working on harvesting machine for automation since 1960s [3,4,5]. Since then, there has been much research undergoing in many countries, such as Japan, America, Netherlands, France [6-14]. While in China, much work on harvesting robot has begun in the late 1990s, and many harvesting robots like the strawberry harvesting robot [15] and the cucumber harvesting robot [16] have been developed. Nevertheless, there are few commercialized harvesting robots, and one of the main problems might be the intelligence of the robots could not meet the demand of the complex environment [17]. Our research group has been focusing on the behavior control and the intelligent simulation system of agricultural harvesting robot based on virtual reality $[5,21-27]$, providing a new research tool to study agricultural harvesting robot.

Aiming at the challenge of intelligent control of harvesting robot, this paper focuses on the control system and simulation system. With a harvesting robot prototype named HNrobot2, the laboratory test and experiments were performed to verify the validity of the harvesting robot and the simulation module.

\section{HARVESTING CONTROL SyStem OF THE HARVESTING ROBOT}

\section{A. The Hardware of the Ccontrol System}

This study went for PC+DSP+FPGA architecture. The overall structure of HNrobot 2 and its system are showed in Figure I. The PC would firstly deal with the data from vision system and send the command to DSP. The DSP would then calculate the parameters of the motors and send them to FPGA which would drive the robot.

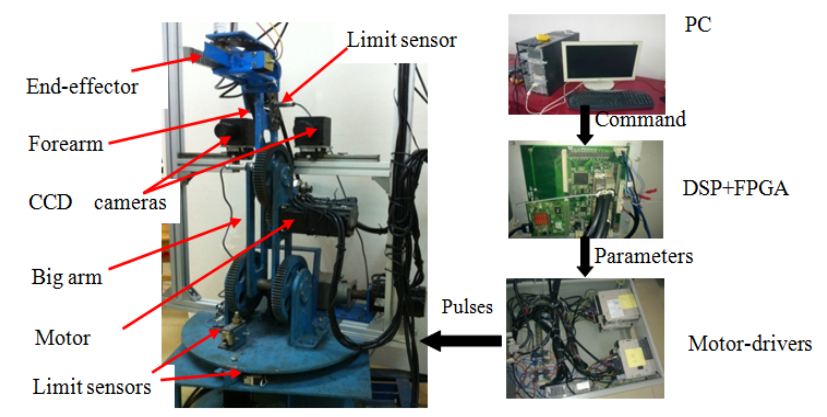

FIGURE I. THE OVERALL STRUCTURE OF HNROBOT2.

\section{B. Behavior Knowledge Database of the Harvesting Robot}

The harvesting behavior is defined as follows: the harvesting behavior is that for the purpose of harvesting fruit objects, it should not damage the fruit and any of its kind, avoid obstacles and reach the given destination; by holding, grasping, cutting or any of this kind, the manipulator could separate the fruit objects from the tree, and finally put them back to baskets.

As can be seen, some basic behaviors could be identified as follows: behavior of communication $(\mathrm{ComBh})$ : data exchange among the behavior controller, sensors and other controller; behavior of standby $(S t a B h)$ : reset the position and orientation of the manipulator to its initial ones; behavior of looking for the target $(L o o k B h)$ : use the sensors such as vision sensors to get the knowledge of itself and the environment, and then locate the position of the targets; behavior of approaching the target $(A p p r B h)$ : the process of taking the end-effector to a position to the target; behavior of obstacle avoidance $(O b A$ voidBh $)$ : the process of obstacle avoidance when approaching to the target; behavior of picking (PickBh): the process of separating the fruit and its stem after the end-effector reaches the harvesting 
position; behavior of collecting (CollBh): the process of collecting the separated fruit back to some basket.

Based on the knowledge model and expression in literature [20], we denote $\mathrm{F}$ (BehaviorID, Bname, input/output) as a behavior for knowledge database, where $\mathrm{F}$ indicates a kind of behavior, BehaviorID indicates identity, Bname is the name of behavior, input and output indicate the input and output parameters. And by establishing the basic behaviors database as shown in table 1 , the foundation for complex behavior could be provided. Behavior knowledge database was established for the behaviors in Table I.

TABLE I. BEHAVIOR KNOWLEDGE DATABASE

\begin{tabular}{|l|c|c|c|}
\hline $\begin{array}{c}\text { Basic } \\
\text { Behavior }\end{array}$ & Behavior ID & ID Functions & Inputs/ Outputs \\
\hline ComBh & 001 & Communication & $*$ \\
\hline StaBh & 002 & Standby & $*$ \\
\hline LookBh & 003 & Finding & $*$ \\
\hline$\ldots$ & $\ldots$ & $\ldots$ & $\ldots$ \\
\hline
\end{tabular}

\section{Basic Model of The Behavior Control}

The basic model is shown in Figure II. $\omega=\{\omega 1, \omega 2, \ldots \omega \mathrm{n}\}$ is the coefficient of the corresponding behaviors, and the value is either 1 when the corresponding behavior is activated, or 0 when it is not activated. $T=\{t 1, t 2 \ldots t \mathrm{n}\}$ is the time sequence coefficient whose value is either 1 or 0 like $\omega . T 1$ is prior to $T 2$, likewise $T 2$ is prior to $T 3$ and so on

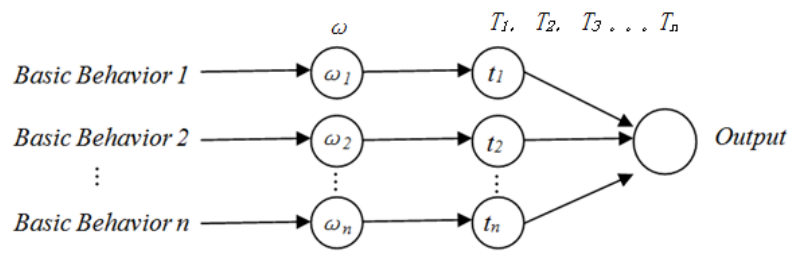

FIGURE II. BASIC MODEL OF THE BEHAVIOR CONTROL.

Firstly, according to the information obtained by vision system and other sensors which is the behavior of looking for the target $(L o o k B h)$, PC would give command that indicate which behaviors in the database are required by sending $\omega$ and Ts. For example, there are 10 basic behaviors in the database, and the required behavior should be a combination of 4 basic behaviors with behavior ID 1, 2, 5 and 8 , and the sequence of

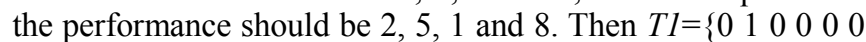

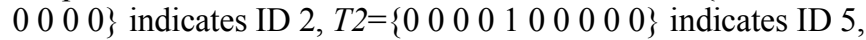

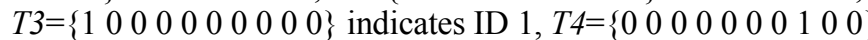

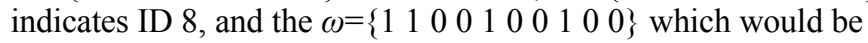
sent to DSP. Secondly, DSP would execute the functions of the corresponding to basic behaviors based on the values of $\omega$ and $T s$. In the example, $\omega_{1}$ of $\omega$ is equal to 1 and $t 2$ of $T 1$ equal to 1 , the output would be the basic behavior with behavior ID 2 which would dominate the DSP and calculate the parameters for the motor drivers. When the behavior finishes, $T 1$ would set itself to all zeros so that $T 2$ would take over for the next basic behavior and so on. When all $T S$ are zeros, the required behavior would be finished.

\section{Vision System}

The vision system is one of the most important components installed in the robot. It helps robots to recognize and locate the targe. It is reported that low positioning accuracy rate and time consumption hinder the developed robots working in a greenhouse or natural environment [2]. To improve the accuracy and reduce the time, a stereo vision experimental platform was proposed to study the virtual manipulator based positioning in VE [5], and the vision system has been developed as shown in Figure III.

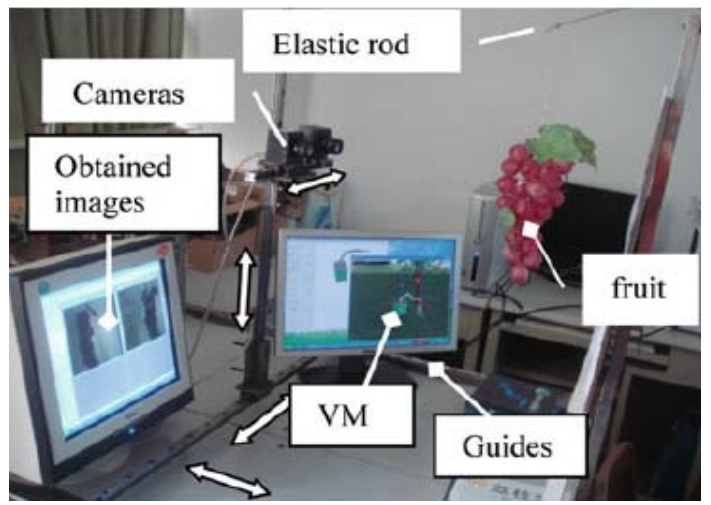

FIGURE III. THE STEREO VISION EXPERIMENTAL PLATFORM AND THE VIRTUAL MANIPULATOR (VM) POSITIONING.

With the vision system, litchi fruit was took as the research object for recognition and positioning as shown in Figure IV. Firstly, according to the color feature of mature litchi, color models such as HSI color model and $\mathrm{YCbCr}$ color model were investigated for image recognition, and $\mathrm{Cr}$ color channel was selected to further segmentation because it could suppress noise and its histogram reveals two peaks for segmentation. Secondly, because of the complex background in the natural environment segmentation algorithms such as Otsu, K-means, FCM and their combination were studied improved the segmentation accuracy among the target fruit, peduncle and background. Finally, locate the best harvest point for the harvesting robot.
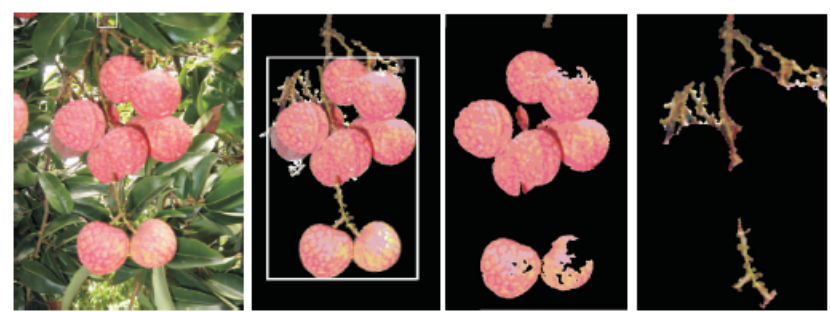

FIGURE IV. THE PROCESS OF IMAGES SEGMENTATION.

It is very difficult to determine the best harvest point for the harvesting robot, because litchi fruit usually grows in bundle and there are obstacles like leaves and tree branch nearby. Therefore, the best harvest point by far is determined as the farthest point on the peduncle from the target fruit based on the image segment results.

\section{The Simulation System of The Behavior Control}

A virtual manipulator positioning simulation system has been developed by combining a simulation module and an 
experimental platform in 2009[5]. In order to further the study of design and simulation, an intelligent simulation system was developed to simulate the behavior control of the harvesting robot in virtual environment (VE) as shown in Figure V. According to the parameters and mathematical model, the simulation module would simulate the behavior control which would drive the virtual harvesting robot, and the simulation data could be validated and later applied to physical prototype.

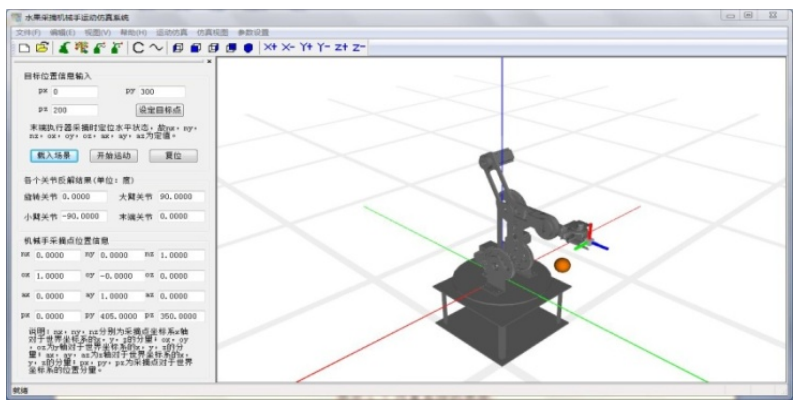

FIGURE V. INTERFACE OF THE SIMULATION SYSTEM.

The simulation module is developed by EON Studio 5.2 and EON SDK. Firstly, 3D models should be built with different software such as AutoCAD, Pro/E or Solidworks. These models would be easily input into VE by the built-in format conversion.

Secondly, make the node settings for all the models and their behaviors in VE. A 'node' is a basic unit in VE, which is an object set of functions and data, including 'field' and 'event', and all the models in VE could be added into different nodes and be managed based on nodes. The 'field' is the attribute values of the node, while the 'event' is a behavior sending or receiving messages among the 'field' of the 'node', which is called event-driven mechanism.

In the simulation module, it is easy to set different motions or behaviors of the models in the virtual environment by setting parameters of nodes, and the parameters could be given beforehand or obtained from the calculation of DSP

\section{PRELIMINARY EXPERIMENT AND RESULTS}

\section{A. Simulation Experiment of the Harvesting Behavior}

The simulation experiment is as follows: firstly, open the simulation module and load a manipulator and obstacles into the visual view window; initialize the setting of the manipulator, the obstacles and the target point; in this experiment, the manipulator, obstacles and the target are set in the plane $\mathrm{XZ}$, and the coordinate of the two obstacles and the target are $(250,250),(350,200)$ and $(600,100)$ respectively with the unit of millimeter. The view after setting is shown in the Figure VI.

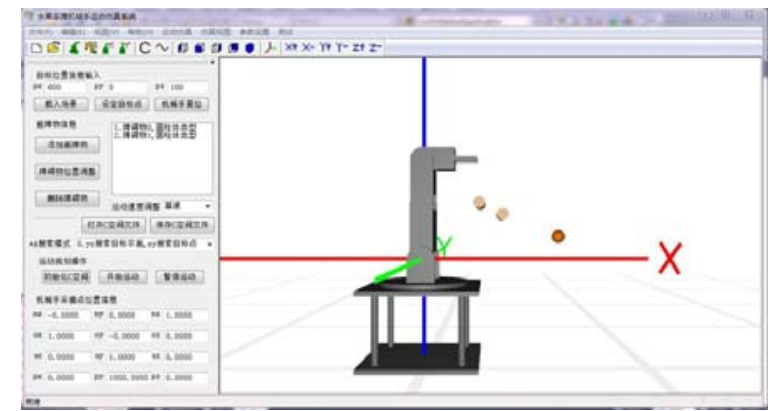

FIGURE VI. THE INTERFACE OF OBSTACLE AVOIDANCE PATH PLANNING MODULE OF VIRTUAL HARVESTING ROBOT.

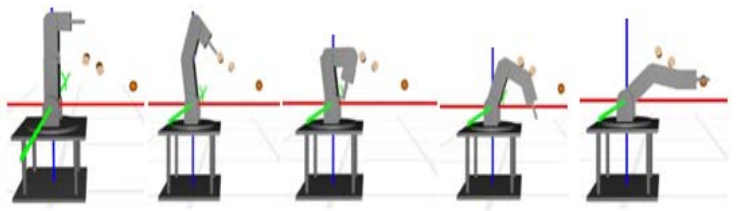

FIGURE VII. THE SIMULATION MOTION PROCESS

The basic behaviors including ComBh, StaBh, LookBh $O b A$ voidBh and PickBh are activated to perform the harvesting behavior which indicates that $\omega=\left\{\begin{array}{lllllll}1 & 1 & 1 & 0 & 1 & 1 & 0\end{array}\right\}, T 1=\left\{\begin{array}{lllll}1 & 0 & 0 & 0 & 0\end{array}\right.$ $0 \quad 0\}, T 2=\left\{\begin{array}{lllllll}0 & 1 & 0 & 0 & 0 & 0 & 0\end{array}\right\}, T 3=\left\{\begin{array}{lllllll}0 & 0 & 1 & 0 & 0 & 0 & 0\end{array}\right\}, T 3=\left\{\begin{array}{lllllll}0 & 0 & 0 & 0 & 1 & 0 & 0\end{array}\right\}$ and $T 4=\left\{\begin{array}{lllllll}0 & 0 & 0 & 0 & 0 & 1 & 0\end{array}\right\}$.

The visualized simulation in VE is shown in Fig 10. The robot would reach the required position at $(600,100)$ and avoid the two obstacles at $(250,250)$ and $(350,200)$.

\section{B. Laboratory Experiment of the Harvesting Behavior}

In laboratory test, a fruit tree was transplanted into our lab and target fruit models were hung on the tree with no obstacle around. Firstly, LookBh behavior would try to find the target with vision system. The vision system module processed the images and obtained the environmental data, which would be sent to PC and set the $\omega$ and Ts coefficients. The robot should be in its initial position before any motion, therefore ComBh and StaBh behavior would make sure the robot is ready to move as shown in Figure VIII (A). Secondly, because of the simple setting of the environment, the PickBh would drive the robot to approach and harvest the target as shown in Figure VIII (B).

As can be seen, the experiment was carried out in a simple condition and deeper research on the behavior control should be further researched; therefore, in the future much more experiments should be carried out including the conditions with obstacles. At the same time, the behavior of the harvesting robot should include the behaviors of moving like a mobile robot. 


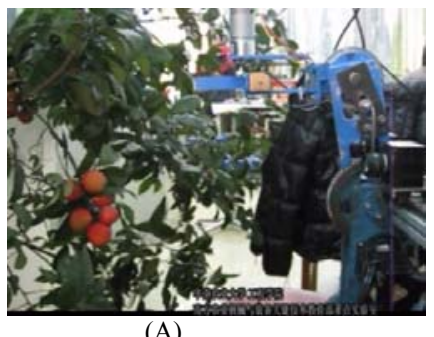

(A)

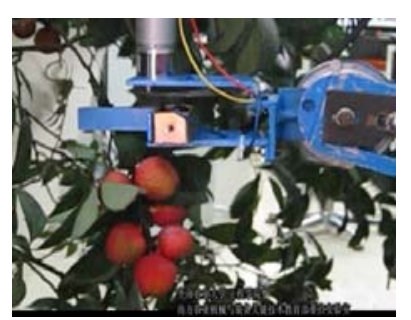

(B)
FIGURE VIII. LABORATORY TEST OF HARVESTING BEHAVIOR CONTROL WITHOUT OBSTACLES: (A) COMBH AND STABH BEHAVIOR; (B) PICKBH BEHAVIOR

\section{CONCLUSIONS AND FutURE RESEARCH}

Aiming at the challenge of intelligent control of harvesting robot, a harvesting robot and its control system was developed. Firstly, the concepts of the harvesting behavior and its basic behaviors were defined, and based on those concepts the behavior knowledge database was established as a library of the functions of the basic behaviors. Secondly, a model of behavior control was proposed including the weight coefficients $\omega$ and the time sequence coefficient $T$ which could provide the possibility of combining the different basic behaviors in the behavior knowledge database. Thirdly, a stereo vision system was developed. Finally, an intelligent simulation system of the behavior control was developed, and the details of setting of the behavior control of the simulation module were described.

Even thought the preliminary experiments were performed and showed the harvesting robot worked functionally well based on the behavior control of the harvesting robot and the software module, further research is needed. Firstly, based on the behavior database, collect more basic behaviors and find an intelligent algorithm to generate new behaviors combining the basic behavior with the simulation system. Secondly, a more advanced robot should be developed with a more compact system, including the control system and the vision system.

\section{ACKNOWLEDGMENTS}

This work is supported by the scholarship of the Guangdong Hopson-Pearl River Education Development Foundation (NO: H2011004), and the National Natural Science Foundation of China (NO: 31171457). Project of Shenzhen science and technology plan (NO: JCYJ20150626102255212, NO: JCYJ20160307101647019).

\section{REFERENCES}

[1] Zhao, D., et al., Design and control of an apple harvesting robot. Biosystems Engineering, 2011. 110(2): p. 112-122.

[2] Libin, Z., et al., Overview of research on agricultural robots in China. International Journal of Agricultural and Biological Engineering. (China), 2008. 1(1): p. $12-21$.

[3] Coppock, G.E., Picking citrus fruit by mechanical means. Florida State Horticultural Society, 1961. 1362.

[4] Li, P., S. Lee and H. Hsu, Review on fruit harvesting method for potential use of automatic fruit harvesting systems. Procedia Engineering, 2011. 23(0): p. $351-366$.
[5] Zou, X., H. Zou and J. Lu, Virtual manipulator-based binocular stereo vision positioning system and errors modelling. Machine Vision and Applications, 2012. 23(1): p. 43-63.

[6] Li, Y., et al., Application and Development Status of China's Agricultural Robot. Tropical Agricultural Engineering, 2014, 38(04): 1315.

[7] Wang, R. and Sun, B, Development Status and Expectation of Agricultural Robot. Bulletin of Chinese Academy of Sciences, 2015, 30(06): 803-809.

[8] Chong, V.K., et al., Development of mobile eggplant grading robot for dynamic in-field variability sensing: Manufacture of robot and performance test. Engineering in Agriculture, Environment and Food, 2008. 1(2): p. $68-76$.

[9] Monta, M., N. Kondo and Y. Shibano. Agricultural robot in grape production system. 1995. New York, NY, USA.

[10] Van Henten, E.J., et al., Optimal manipulator design for a cucumber harvesting robot. Computers and Electronics in Agriculture, 2009. 65(2): p. 247-257.

[11] Van Henten, E.J., et al., An autonomous robot for de-leafing cucumber plants grown in a high-wire cultivation system. Biosystems Engineering, 2006. 94(3): p. 317-323.

[12] Van Henten, E.J., et al., Field Test of an Autonomous Cucumber Picking Robot. Biosystems Engineering, 2003. 86(3): p. 305-313.

[13] Van Henten, E.J., et al., An autonomous robot for harvesting cucumbers in greenhouses. Autonomous Robots, 2002. 13(3): p. 241-258.

[14] Bourely, A. and J.M. Roger. A knowledge-based system for the control of a citrus harvesting robot. 1993. Nanterre, France.

[15] Feng, Q., et al., New strawberry harvesting robot for elevated-trough culture. International Journal of Agricultural and Biological Engineering, 2012. 5(2): p. 1-8.

[16] Ji, C., et al., Development and Performance Analysis on Cucumber Harvesting Robot System in Greenhouse. Robot, 2011. 33(6): p. 726-730. (Chinese)

[17] Chen, D., Chen, J., and Ma, X.. Key technologies of the agricultural robot and its research progress. Journal of Agricultural Mechanization Research, 2010(5): p. 207-211

[18] Zou, X., et al., Modeling for Behavior and Simulation of Agriculture Mobile Robot in Virtual Environment. Journal of System Simulation, 2006. 18(2): p. 551-553,562.

[19] Zou, X., et al., Knowledge Modeling of harvesting behaviors of the Virtual mobile robot. Equipment Manufactring Technology, 2007(01): p. 57-59, 63.

[20] Zou, X., et al., Research on Picking Manipulator Virtual Design and Simulation System. Journal of System Simulation, 2010(11): p. 27482752.

[21] Zou, X., et al., The experimental platform of positioning associated with machinery and vision, Chinese Patent, ZL200810027369.6,2009.11

[22] Zou, X., et al., Modelica-based Modeling Research of Picking Manipulator Motion Control Process. Journal of Simulation Sytem, 2009(18): p. 5882-5885.

[23] Zou X., et al.(2012).Multi-fruits and vegetable harvesting robot and its end effector, Chinese Patent, ZL201010565235.7

[24] Zou, H., et al. Real-time simulation system of virtual picking manipulator based on parametric design. in 2nd International Conference on Intelligent Robotics and Applications, ICIRA 2009, December 16, 2009 - December 18, 2009. 2009. Singapore, Singapore: Springer Verlag.

[25] Zou, X., et al., Research on manipulator positioning based on stereo vision in virtual environment. Key Engineering Materials, 2009. 392-394: p. 200-204.

[26] Zou, H., et al. Research on virtual design and simulation system for manipulators of multi multi-degree of freedom. in 2009 International Conference on Manufacturing Science and Engineering, ICMSE 2009, December 26, 2009 - December 28, 2009. 2010. Zhuhai, China: Trans Tech Publications.

[27] Chen, Y., et al., Development of the picking manipulator simulation platform based on multi-domain system, in Advanced Materials Research. 2010: Hangzhou, Zhejiang, China. p. 292-296. 